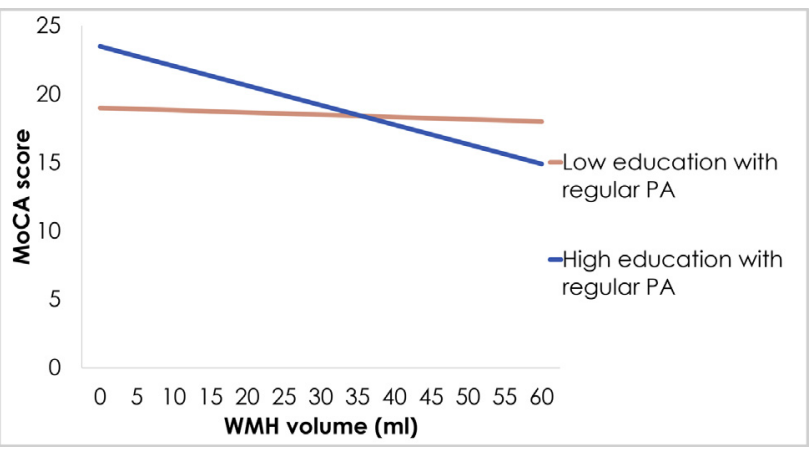

TIA. Cognitive functions were measured using the Montreal Cognitive Assessment (MoCA). Multiple linear regression analysis was conducted to explore the associations between leisure activity participation with $\mathrm{WMH}$ and the moderating effects of leisure activities upon relationship between WMH and MoCA (with respective activity $\mathrm{x} \mathrm{WMH}$ volume interaction). Analyses were further stratified by low ( $<6$ years) or high education ( $\geq 6$ years). Effect of aerobic and non-aerobic physical activities was also compared. All models were adjusted with age, sex and years of education. Results: PA, but not IA, was negatively related to $\mathrm{WMH}$ volume $(\mathrm{b}=-$ $3.45, \mathrm{p}<.05)$. IA $(\mathrm{b}=3.81, \mathrm{p}<.001)$ contributed to the MoCA scores. Only IA, but not PA, has main effect towards MoCA score after stroke/TIA $(b=3.81, p<.001)$. Significant interaction with $\mathrm{WMH}$ volume was found for PA $(\mathrm{b}=0.27, \mathrm{p}<.01)$ but not with IA. Such interaction was found in the lower education group $(\mathrm{b}=.28, p<.01)$ but not in the higher education group. Conclusions: In patients with stroke or TIA, IA confers general cognitive benefits. Regular participation of PA negatively correlated with patients WMH volume and in patients with low education, cognitive benefits of PA increases with severity of WMH.

\section{P2-575 APOE EPSILON 4 STATUS MODIFIES THE EFFECTS OF AEROBIC, RESISTANCE, OR COMBINED TRAINING ON COGNITIVE FUNCTION IN OLDER ADULTS}

Taeko Makino ${ }^{1}$, Hiroyuki Umegaki ${ }^{1}$, Takahiro Hayashi ${ }^{1}$, Xian Wu Cheng ${ }^{1}$, Hiroyuki Shimada ${ }^{2}$, Hiroko Dodge ${ }^{3,4}$, Masafumi Kuzuya ${ }^{1},{ }^{1}$ Nagoya University, Nagoya, Japan; ${ }^{2}$ National Center for Geriatrics and Gerontology, Obu, Japan; ${ }^{3}$ University of Michigan, Ann Arbor, MI, USA; ${ }^{4}$ Oregon Health \& Science University, Portland, OR, USA. Contact e-mail: tmakino@med.nagoya-u.ac.jp

Background: It has been suggested that the presence of an APOE $\varepsilon 4$ allele is associated with a higher risk for Alzheimer's disease. Physical exercise is known to be beneficial for preventing cognitive decline in older adults. The interactive effects of APOE status and physical exercise on cognitive function have not been fully investigated. In the "TOyota Prevention Intervention for Cognitive decline and Sarcopenia (TOPICS)", we used a randomized controlled trial design to investigate differential effects of exercise modalities on cognitive function among community-dwelling older adults. We examined the relationship between APOE status and differential physical exercise in these intervention programs. Methods: A total of 415 subjects aged $65-85$ years who met our inclusion and exclusion criteria were randomly assigned to one of the following 6-month, twice-per-week programs: (1) aerobic training (AT), (2) resistance training (RT), (3) combined training (CT), or no training (control group). Our primary outcome was memory function (the Logical Memory II delayed recall scores [LM II]). We assessed cognitive function via a battery of neuropsychological tests. The APOE status was classified based on the results of the immunoblot technique using isoelectric focusing. We used a repeated-measures analysis of variance (ANOVA) to evaluate the effect of the interventions. For stratified analysis according to the APOE status, we divided the cohort into 2 groups: APOE $\varepsilon 4$ carrier and non-carrier. We compared differences in the outcome measures in APOE $\varepsilon 4$ stratified patients. Results: Overall, the repeated-measures ANOVA revealed a main effect of time $(\mathrm{F}(1,374)=70.57, \mathrm{p}<0.001)$ and indicated an improvement in LM II performance compared with baseline after the intervention. However, we did not find any significant interactions for group $\times$ time with respect to the LM II. In APOE $\varepsilon 4$ carrier group $(\mathrm{n}=75)$, we found a significant group $\times$ time interaction for the LM II $(\mathrm{F}(3,71)=3.42, \mathrm{p}=0.02)$. The LM II significantly improved in the AT group and the RT group compared with the control group. No significant group interactions with the LM II were observed in the APOE $\varepsilon 4$ non-carrier group $(n=303)$. Conclusions: Aerobic or resistance training interventions may improve memory function among communitydwelling older adults with APOE $\varepsilon 4$.

\section{P2-576 BRAIN PLASTICITY FOLLOWING PHYSICAL TRAINING IN INDIVIDUALS WITH MILD COGNITIVE IMPAIRMENT: NEUROIMAGING STUDY}

Yulia Lerner $^{1,2}$, Galit Yogev-Seligmann ${ }^{1}$, Tamir Eisenstein ${ }^{1,2}$, Elissa Ash ${ }^{1}$, Talma Hendler ${ }^{1,2}$, Nir Giladi ${ }^{1,2},{ }^{1}$ Tel Aviv Sourasky Medical Center, Tel Aviv, Israel; ${ }^{2}$ Tel Aviv University, Tel Aviv, Israel. Contact e-mail: yulia.lerner@ gmail.com

Background: Patients with Alzheimer's disease (AD) pass first through a stage of mild cognitive impairment (MCI), where milder neuropsychological deficits are detectable, usually but not always in the memory domain. The amnestic subtype (a-MCI) is characterized by impairment in information and memory processing. Previous studies in animals suggested that physical training results in structural and functional brain changes. However, the extent to which comparable improvement can be found in individuals with a-MCI are unknown. Our recent study revealed that processing of real life information in participants with a-MCI shifted from the traditional high-level perceptual and cognitive areas toward the pre-central and central sulci. Here, we evaluate how information processing as well as memory encoding might be modulated by physical training in a-MCI. Methods: Neuropsychological evaluations, assessment of neurotrophic factor (BDNF), cardiorespiratory fitness assessment and fMRI experiments have been performed before the beginning of physical training (aerobic or non-aerobic) and following the intervention. 24 participants suffering of a-MCI carried out their activity routines $3 \mathrm{~d} / \mathrm{wk}$ for 45 -min session for 4 months under supervision of an experienced trainer. Neuropsychological memory tests evaluated short term memory and retention of information. During fMRI sessions participants were presented with a narrated real-life story and a free recall paradigm that compared the acquisition of novel vs. over-learned stimuli. Several analytic approaches have been used for data analysis depending on 